\title{
Model independent constraints on the cosmological expansion rate
}

\author{
Edvard Mörtsell ${ }^{1}$ and Chris Clarkson ${ }^{2}$ \\ ${ }^{1}$ Department of Physics, Stockholm University, AlbaNova University Center \\ S-106 91 Stockholm, Sweden \\ ${ }^{2}$ Cosmology and Gravity Group, Department of Mathematics and Applied \\ Mathematics, University of Cape Town, Rondebosch 7701, Cape Town, South Africa \\ E-mail: edvard@physto.se, chris.clarkson@uct.ac.za
}

\begin{abstract}
We investigate what current cosmological data tells us about the cosmological expansion rate in a model independent way. Specifically, we study if the expansion was decelerating at high redshifts and is accelerating now, without referring to any model for the energy content of the universe, nor to any specific theory of gravity. This differs from most studies of the expansion rate which, e.g., assumes some underlying parameterised model for the dark energy component of the universe. To accomplish this, we have devised a new method to probe the expansion rate without relying on such assumptions.

Using only supernova data, we conclude that there is little doubt that the universe has been accelerating at late times. However, contrary to some previous claims, we can not determine if the universe was previously decelerating. For a variety of methods used for constraining the expansion history of the universe, acceleration is detected from supernovae alone at $>5 \sigma$, regardless of the curvature of the universe. Specifically, using a Taylor expansion of the scale factor, acceleration today is detected at $>12 \sigma$. If we also include the ratio of the scale of the baryon acoustic oscillations as imprinted in the cosmic microwave background and in the large scale distribution of galaxies, it is evident from the data that the expansion decelerated at high redshifts, but only with the assumption of a flat or negatively curved universe.
\end{abstract}

Keywords: dark energy theory, supernova type Ia

\section{Introduction}

It is becoming generally acknowledged that the observed redshift-distance relation from Type Ia supernovae (SNe Ia), together with the scales of baryon acoustic oscillations as observed in the distribution of galaxies on large scales (BAO) and the temperature anisotropies in the cosmic microwave background (CMB), implies that the current energy density in the universe is dominated by dark energy, here defined as a component with an equation of state, $w=p / \rho<-1 / 3$. Current cosmological data are consistent with the standard - or concordance - cosmological model, where $\Omega_{m}=0.3$ and $\Omega_{\Lambda}=0.7$, i.e., the dark energy is explained in terms of a cosmological constant or vacuum energy with $w=-1$ [e.g., 1, 11, 15, 16, 24, 35]. For the concordance model, the universal 
expansion is accelerating at redshifts lower than $z \sim 0.7$ and decelerating at higher redshifts.

Most analyses which attempt to infer something about the expansion history of the universe rely on a specific model, e.g., a dark energy 'fluid' or a modification of general relativity, which has one or two parameters of interest [13. Consequently, much of our knowledge of the expansion history of the universe has these parameterisations hardwired into our conclusions, which may leave a large space of possible expansion histories unexplored. Alongside these analyses, it is therefore constructive to try to assert model independent statements where we can. The question we address in this paper is to what extent we can infer changes in the expansion rate without referring to any theory of gravity or model for the energy content of the universe. That is; what is the history of the universal expansion?

Unfortunately, it is difficult to give a definite answer to this question since two important assumptions are implicit in all discussion of this kind. It is known that spherically symmetric void - or Hubble bubble - models may explain the anomalous Hubble diagram whilst always maintaining a decelerating expansion rate, at the price of violating the Copernican principle [3, 4, 6, 10, 18, 19, 20, 38, 46, 48,. Ideally we should test the Copernican principle in a model independent way [8, 45], and so rule these models out. A further assumption in the standard model - insofar as determining the expansion dynamics is concerned - is that that the universe is smooth enough at small distances to be described by a perfectly homogeneous and isotropic model [2, 5, 12, 26, 27, 28, 32, 33, 37, 41]. At best this gives a small error to all our considerations; at worst, many of our conclusions might be wrong.

Since it was traditionally thought that the expansion rate would be decelerating, we measure acceleration with the deceleration parameter, $q$, defined by

$$
q \equiv-\frac{\ddot{a} a}{\dot{a}^{2}}=-\frac{\ddot{a}}{a H^{2}},
$$

where $a$ is the scale factor, dots denote derivatives with respect to time and the Hubble parameter, $H$, is defined as $H \equiv \dot{a} / a$. Because of the sign convention, negative values for $q$ correspond to acceleration. Traditionally, the goal when observing the expansion of the universe was to constrain two parameters, the current values of the Hubble parameter - or the Hubble constant $-H_{0}$, and the deceleration parameter, $q_{0}$. The first signs of an accelerated expansion came 10 years ago with the observations that distant SN Ia appear dimmer than expected in a universe with constant or decelerated expansion velocity [30, 34, 39]. However, the acceleration was only evaluated in terms of a model with $\Omega_{m}$ and $\Omega_{\Lambda}$, in which $q_{0}=\Omega_{m} / 2-\Omega_{\Lambda}$, see Eq. (4).

In 2002, Turner and Riess [44] studied the change in the expansion velocity without referring to the energy content or theory of gravity - by using a step model for the deceleration parameter where $q$ had one constant value at low and intermediate redshifts and another constant value at high redshifts. They demonstrated, that the SN Ia data at the time showed a strong preference for acceleration today and deceleration in the past, if the transition redshift was set (by hand) to $z=0.4-0.6$. However, this 
conclusion relied heavily on the observed magnitude of a single supernova, SN1997ff, the most distant SN Ia observed at $z=1.755$. Being very distant, it is especially susceptible to systematic effects that may reduce its cosmological utility. One such effect is gravitational lensing that has been shown to brighten SN1997ff by $\sim 0.15$ magnitudes [23].

Also, in Shapiro and Turner [43], it was shown that marginalising over the transition redshift, considerably relaxed the constraints on the expansion history. Specifically, using the so called "gold" dataset consisting of 157 SNe Ia [36], the authors only found strong evidence for acceleration at some epoch, not necessarily at $z<0.1$, and that $q$ was higher in the past.

In 2004, Riess et al. [36], the gold dataset was used to constrain a Taylor expansion of $q(z)$,

$$
q(z)=q_{0}+z q^{\prime}(z=0),
$$

where the prime denotes a derivative with respect to redshift. The result found was that $q_{0} \lesssim-0.3$ and $q^{\prime} \gtrsim 0$ at $95 \%$ confidence level (CL). The evidence that $q^{\prime} \gtrsim 0$, was interpreted as evidence for deceleration at higher redshifts. However, the Taylor expansion should only be meaningful for $z<1$, up to which the parameter space $\left[q_{0}, q^{\prime}\right]$ still allows for acceleration at $95 \%$ CL. Also, the fit is done using data at $z>1$, for which higher order terms in the Taylor expansion should be important.

In, Elgarøy and Multamäki [17], the same data was found to be consistent with a constant negative deceleration parameter, and in Rapetti et al. [31, the SN data was combined with X-ray cluster gas mass fraction measurements to constrain the deceleration parameter, $q$, as well as the next order derivative of the scale factor as decoded in the jerk parameter, $j$. See also Daly et al. [14] for an alternative approach to constraining the acceleration history of the universe.

In this paper, we follow the spirit of previous work, in that we strive to make as few assumptions as possible regarding the theory of gravity or the energy content of the universe when inferring the state of the expansion velocity of the universe. In fact, the only assumptions used in this paper, other than those mentioned, is that SNe Ia are standardisable candles and that the observed inhomogeneities in the large scale distribution of galaxies and the anisotropies in the temperature of the CMB reflects the same physical scale. One feature we discuss in particular is the role of curvature in determining the acceleration, as there are significant degeneracies between curvature and acceleration, in a similar vein which exists between curvature and the dark energy equation of state $w$ [9, 21].

In Sec. 2, we discuss acceleration and deceleration within the standard model, as well as possible observational measures of the deceleration parameter. In Sec. 3, we present the two sources of data used in this paper, and in Sec. 4, we present a new method for inferring the expansion history of the universe, together with our results for $q(z)$. Our results are summarised in Sec. 5. In short, we conclude that the evidence for late time acceleration of the universal expansion is very strong, regardless of the method 


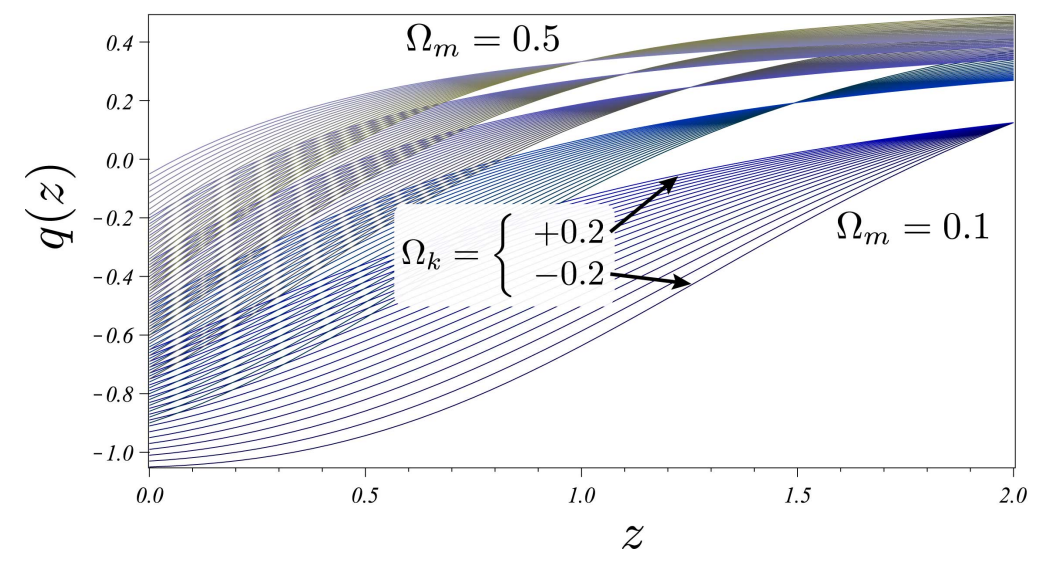

Figure 1. The range of behaviour available to $q(z)$ for the $\Lambda$ CDM model.

used to measure $q(z)$, whereas deceleration at high redshifts can still be avoided given current data.

\section{Acceleration and deceleration}

First, let us calculate what to expect for $q(z)$ in the standard $\Lambda$ CDM model, with matter density, $\Omega_{m}$, and a cosmological constant, $\Omega_{\Lambda}=1-\Omega_{m}-\Omega_{k}$. The acceleration equation is given by

$$
\frac{\ddot{a}}{a}=-\frac{4 \pi G}{3}(\epsilon+3 p)=-\frac{4 \pi G}{3}\left(\rho_{m}-2 \rho_{\Lambda}\right) .
$$

This gives

$$
q(z)=\frac{1}{2} \frac{\Omega_{m}(1+z)^{3}-2\left(1-\Omega_{m}-\Omega_{k}\right)}{\Omega_{m}(1+z)^{3}+\Omega_{k}(1+z)^{2}+1-\Omega_{m}-\Omega_{k}} .
$$

In the infinite future $(z=-1)$, we have $q=-1$, and as $z \rightarrow \propto$ f $q=0.5$. The transition redshift between acceleration and deceleration is given by $z_{t}=\left[2\left(1-\Omega_{m}\right) / \Omega_{m}\right]^{1 / 3}-1$ when $\Omega_{k}=0$. For the flat concordance cosmology with $\Omega_{m}=0.3$, we have $z_{t} \sim 0.7$. One of the main problems we have in determining acceleration as a function of redshift, is the wide range of behaviours $q(z)$ can exhibit, especially if curvature is present. In Fig 1 , we show the range of values $q(z)$ can take on within the $\Lambda$ CDM paradigm by varying the constants $\Omega_{m}$ and $\Omega_{k}$. With more exotic dark energy models the variation can be much more elaborate and $q(z)$ can be regarded as a free function to be constrained by observations. Then, once $q(z)$ is determined, the dark energy equation of state, $w(z)$, can in principle be determined from a first-order differential equation. However, since the integration constant (i.e., $H_{0}$ ), as well as $\Omega_{k}$ and $\Omega_{m}$ are arbitrary, there is a three parameter family of dark energy models which can give rise to the same $q(z)$.

$\ddagger$ In fact, this approximation is only valid after the universe became matter dominated at $z \sim 3000$. 
Normalising the scale factor to be unity today, $a=(1+z)^{-1}$, we may derive $\dot{z}=-(1+z) H$, and

$$
q(z)=-\frac{\dot{H}}{H^{2}}-1=\frac{H^{\prime}}{H}(1+z)-1=(1+z)\left[\ln \frac{H}{1+z}\right]^{\prime} .
$$

In principle, Eq. (5) can be used to obtain the value of the deceleration parameter as a function of redshift. Unfortunately, it is very difficult to measure the Hubble parameter, $H(z)$, let alone its derivative. As an example, from Fig. 3, it can be understood why it is difficult to differentiate noisy SN Ia data in order to obtain $H(z)$. Note however that with future BAO data, it may be possible to measure $H(z)$ directly [42].

Rearranging Eq. (5), we can write

$$
\frac{H^{\prime}}{H}=\frac{1+q(z)}{1+z} \rightarrow \int_{z_{1}}^{z_{2}} \frac{d(\ln H)}{d z} d z=\int_{z_{1}}^{z_{2}} \frac{1+q(z)}{1+z} d z .
$$

If the universe is accelerating between redshifts $z_{1}$ and $z_{2}$, we have

$$
\ln \left(\frac{H_{2}}{H_{1}}\right)<\ln \left(\frac{1+z_{2}}{1+z_{1}}\right) \rightarrow \frac{\left(1+z_{2}\right)}{H_{2}}>\frac{\left(1+z_{1}\right)}{H_{1}} .
$$

When we have acceleration, $H(z)$ thus grows with redshift faster than $(1+z)$. This is easy to understand since if $\dot{a}=H /(1+z)$ is increasing with redshift, $\dot{a}$ is decreasing with time and $\ddot{a}<0$, corresponding to acceleration. For deceleration, $H(z)$ grows slower than $(1+z)$. For a matter dominated universe, $H=H_{0} \sqrt{\Omega_{m}(1+z)^{3}} \propto(1+z)^{1.5}$, i.e., the expansion is decelerating. For an empty universe, $H=H_{0} \sqrt{\Omega_{k}(1+z)^{2}} \propto(1+z)$, i.e., the expansion velocity is constant. Note however that an empty universe is not the only possible solution for a constant expansion velocity - any universe where the total energy density scales as $(1+z)^{2}$ also gives a constant expansion.

The comoving coordinate distance is given by

$$
d_{c}(z)=\int_{0}^{z} \frac{d z}{H(z)}=\frac{1}{H_{0}} \int_{0}^{z} \exp \left[-\int_{0}^{v} \frac{[1+q(u)] d u}{(1+u)}\right] d v,
$$

and the luminosity distance, which is the relevant quantity for SN Ia observations, is given by

$$
d_{L}(z)=\frac{1+z}{H_{0} \sqrt{-\Omega_{k}}} \sin \left[\sqrt{-\Omega_{k}} H_{0} d_{c}(z)\right] .
$$

The angular diameter distance, relevant for the scale of BAO and $\mathrm{CMB}$, is given by $d_{A}=d_{L} /(1+z)^{2}$. Although having the same constant expansion rate, we would therefore measure different luminosity and angular diameter distances in the flat and open nonaccelerating cases. Determining acceleration at a given redshift is then the same as determining if the function $\left[(1+z) D^{\prime} / H_{0} \sqrt{1-\left|\Omega_{k}\right| D^{2}}\right]$, where $D(z)=d_{L}(z) H_{0} /(1+z)$, is an increasing function of redshift.

\section{Data}

In the last decade, there has been a formidable progress in using cosmological data to constrain the expansion history and the energy content of the universe. Observations 
include, but are not restricted to, SNe Ia, BAO, CMB, weak gravitational lensing and galaxy cluster number counts. In this paper, we make use of SN Ia data together with a combination of CMB and BAO observations that only depends on the expansion history of the universe, not the energy content.

\subsection{Type Ia supernova data}

The Union08 data set 25] is a compilation of SNe Ia from, e.g., the Supernova Legacy Survey, ESSENCE survey and HST. After selection cuts, the data set amounts to 307 SNe Ia, spanning a redshift range of $0 \lesssim z \lesssim 1.55$, analysed in a homogenous fashion using the spectral-template-based fit method SALT.

\subsection{Baryon acoustic oscillations and the cosmic microvawe background}

The distances measured to the CMB decoupling epoch at $z_{*} \sim 1090$ and to the BAO at $z=[0.2,0.35]$ depend on the physical scale of the acoustic oscillations at decoupling, and thus on the matter and baryon density. Specifically, the position of the first peak in the CMB power spectrum, which represents the angular scale of the sound horizon at decoupling, is given by,

$$
l_{\mathrm{a}} \approx \pi \frac{d_{A}\left(z_{*}\right)\left(1+z_{*}\right)}{r_{\mathrm{s}}\left(z_{*}\right)}
$$

where the comoving sound horizon at recombination,

$$
r_{\mathrm{s}}\left(z_{*}\right)=\int_{z_{*}}^{\infty} \frac{c_{s}(z)}{H(z)} d z
$$

depends on the speed of sound, $c_{s}$, in the early universe. The observed scale of the BAO is given by $r_{\mathrm{s}}\left(z_{*}\right) / D_{V}$, where the so called dilation scale, $D_{V}$, is combined from angular diameter and radial distances according to

$$
D_{V}(z)=\left[(1+z)^{2} d_{A}^{2} \frac{c z}{H(z)}\right]^{1 / 3} .
$$

Since we want to infer the expansion history with minimal assumptions regarding the energy density, we take a conservative approach and use the ratio of the observed scales in the $\mathrm{CMB}$ and the BAO. This ratio does not depend on the physical size of the sound horizon at decoupling but only on the assumption that the BAO and CMB reflects the same physical size. Percival et al. 29] derives

$$
\begin{aligned}
& \frac{d_{A}\left(z_{*}\right)\left(1+z_{*}\right)}{D_{V}(z=0.2)}=19.04 \pm 0.58 \\
& \frac{d_{A}\left(z_{*}\right)\left(1+z_{*}\right)}{D_{V}(z=0.35)}=10.52 \pm 0.32 .
\end{aligned}
$$

The measurements (from the 2dFGRS and SDSS, respectively, combined with 3 year WMAP data) are correlated with correlation coefficient $\rho=0.39$. Using WMAP 5 year data instead of 3 year data gives close to identical results, when combined with the BAO data. 


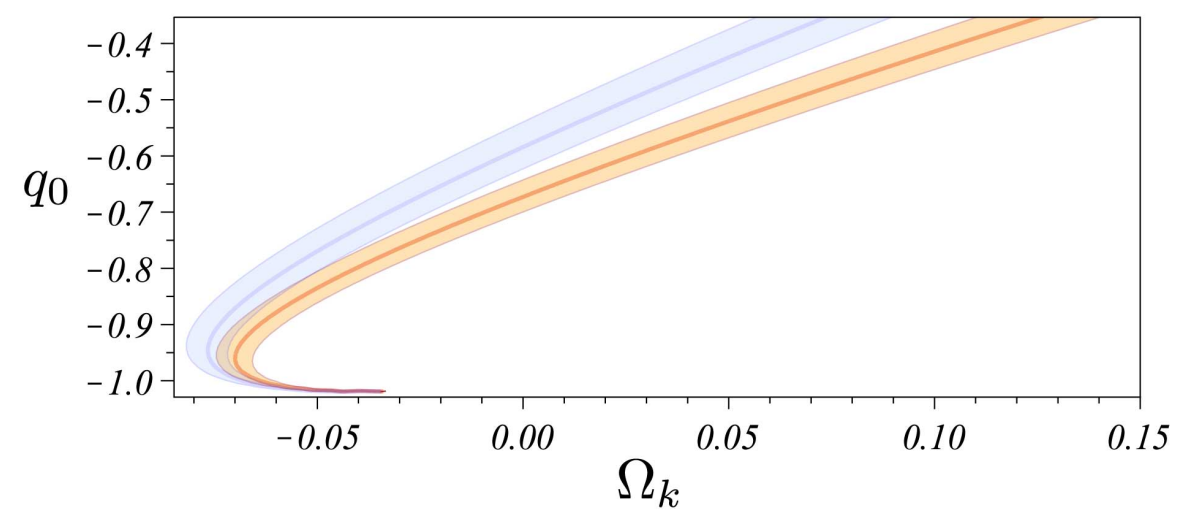

Figure 2. Best fit values of $d_{A}\left(z_{*}\right) / D_{V}(z)$ at $z=0.2$ (red) and $z=0.35$ (blue), together with their $1 \sigma$ errors, as measured from the scale imprinted in the CMB and $\mathrm{BAO}$, shown in the parameter space for $\Lambda \mathrm{CDM}$.

The constraints given by Eq. (13), individually place very weak constraints on acceleration. Also, these constraints are highly degenerate with the curvature. In Fig. 2, we show the $\mathrm{CMB} / \mathrm{BAO}$ constraints in the $\Omega_{k}-q_{0}$ parameter space for the $\Lambda \mathrm{CDM}$ model, with their $1 \sigma$ errors. It is clear that they require a large, negative, deceleration parameter to be consistent with each other at $1 \sigma \mathrm{CL}$, irrespective of the curvature. Note also that a flat model is ruled out at this level. At $2 \sigma$ CL however, individual constraints on $q_{0}$ and $\Omega_{k}$ becomes very weak, and the combined constraints are limited by the strong degeneracy exhibited between $\Omega_{k}$ and $q_{0}$. Nevertheless, the data unambiguosly shows that $\Omega_{k}>-0.1$, i.e., the universe is not strongly overclosed.

\section{Methods and results}

In this section, we discuss how to determine the change of the expansion rate, or $q(z)$, without assuming a model for the energy content of the universe or a specific gravitational theory. We are thus limited to using geometrical data as opposed to methods that are sensitive to the growth of structure in the universe.

First, we discuss some difficulties that occur when trying to find $q(z)$ by comparing observed magnitudes of SNe Ia directly. This method has the advantage that it can, in principle, detect a change in the expansion rate without somewhat ad-hoc parameterisations of $q(z)$. Some of these difficulties have been realised by Seikel and Schwarz [41] - in particular the reliance on low redshift SNe Ia.

Alternatively we may parametrise the expansion with piecewise, constant accelerations, i.e., we assume that $q(z)$ can vary between redshift bins, but is constant within the bins. This is the approach introduced by Turner and Riess [44. We investigate this further below, and find that it suffers from strong degeneracies with curvature when including CMB data. We also investigate alternative parameterisations of $q(z)$, displaying the same degeneracies. However, we show that SN Ia data alone, 
show that $q(z=0)<0$, irrespective of curvature.

We then present a method for determining acceleration, first employed in Riess et al. [35, that relies on the fact that $\dot{a}^{-1}=(1+z) / H(z)$ is an increasing function when the expansion is accelerating which seems to the case at low redshifts. However, since the method relies on differentiating noisy SN Ia data, results at high redshifts have very large uncertainties. Finally we present a new method which relies on a Taylor expansion around an arbitrary 'sliding' redshift.

\subsection{Inferring acceleration from $m(z)$}

In SN Ia cosmology, the validity of a given cosmological model is tested by comparing observed peak SN Ia magnitudes with the theoretical magnitudes for the given model,

$$
m(z)=M+5 \log _{10}\left[\frac{d_{L}(z)}{1 \mathrm{Mpc}}\right]+25
$$

where $M$ is the absolute $\mathrm{SN}$ Ia magnitude. The normalisation of the magnitude (containing, e.g., $M$ and $H_{0}$ ) is usually marginalised over, and constraints are derived by examining the redshift evolution of the magnitudes.

Often, one presents SN Ia data in the form of the difference between the observed peak magnitudes, and the theoretical magnitudes in an empty universe, $m_{e}$, where the difference is normalised to be zero at low redshifts. Since an empty universe is neither accelerating nor decelerating, it is sometimes claimed that one can infer the state of the universal expansion from a quick visual inspection of this difference. One common claim (at talks and discussions, if not in papers) is that negative values of this difference, $m-m_{e}<0$, shows that the universe is decelerating and positive values that it is accelerating. Another inconsistent, but nevertheless common claim, is that if the difference increases with redshift, the universal expansion is accelerating. If the difference is decreasing, the expansion is decelerating. That is, the claim is that we can infer the state of the expansion velocity by studying the sign of the derivative of the difference with respect to redshift. Let us investigate these claims.

For the non-accelerating case, $H \propto(1+z)$, and

$$
d_{c, n}=\frac{1}{H_{0}} \ln (1+z)
$$

and

$$
d_{L, n}=\frac{1+z}{H_{0} \sqrt{-\Omega_{k}}} \sin \left[\sqrt{-\Omega_{k}} \ln (1+z)\right],
$$

where subscript $n$ refers to non-accelerating expansion. For a flat, non-accelerating, universe

$$
d_{L, f}=\frac{1+z}{H_{0}} \ln (1+z) .
$$

For an empty, non-accelerating, universe, $\Omega_{k}=1$, and

$$
d_{L, e}=\frac{1+z}{H_{0}}\left(1+z-\frac{1}{1+z}\right)=\frac{z(z+2)}{H_{0}} .
$$


For small curvature $\left(\Omega_{k} \ll 1\right)$, defining $x=\sqrt{-\Omega_{k}} d_{c}$, we can use a Taylor expansion in $x$ and write

$$
\begin{aligned}
\frac{H_{0} d_{L}}{1+z} & =\frac{1}{\sqrt{-\Omega_{k}}} \sin (x) \\
& =\frac{1}{\sqrt{-\Omega_{k}}}\left(x-\frac{x^{3}}{6}\right)+\mathcal{O}\left(x^{5}\right) \\
& \simeq H_{0}\left(d_{c}+\frac{\Omega_{k}}{6} d_{c}^{3}\right) .
\end{aligned}
$$

The difference between the observed SN Ia magnitudes and those expected in a nonaccelerating universe is given by

$$
\Delta m \equiv m-m_{n}=\frac{5}{\ln 10} \ln \left(\frac{d_{L}}{d_{L, n}}\right) .
$$

In Seikel and Schwarz [40], the inequality (valid in a flat universe)

$$
\begin{aligned}
d_{L}(z) & =\frac{(1+z)}{H_{0}} \int_{0}^{z} \exp \left[-\int_{0}^{v} \frac{[1+q(u)] d u}{(1+u)}\right] d v \\
& <\frac{(1+z)}{H_{0}} \int_{0}^{z} \frac{d z}{(1+z)}=\frac{(1+z)}{H_{0}} \ln (1+z),
\end{aligned}
$$

was considered as evidence $(\sim 5 \sigma \mathrm{CL})$ for some period of acceleration up to redshift $z$, i.e., $q<0$ at some redshift. Allowing for spatial curvature, the evidence becomes significantly weaker, or $1.8 \sigma$. We note [Eq. (18)], that this is equivalent to studying the sign of $\Delta m$, if the universe is flat. Assuming we can get rid of the dependence on $H_{0}$ and $M$ by normalising the difference to be zero at low redshifts, we can study the dependence of the curvature term by looking at the difference when subtracting empty and flat non-accelerating cosmologies (Fig. 3). It is obvious that $\Delta m$ in fact is positive for $z \sim 0.5$, regardless of the curvature of the universe. A similar comparsion between SNe Ia at low and mid/high redshifts was recently used in Seikel and Schwarz [41] to provide a calibration-independent test of the accelerated expansion of the universe, the conclusion being that the universe has accelerated at some epoch at $\sim 4 \sigma$ CL. From Eq. (23), it is evident that the sign of $\Delta m$ only tells us whether the integrated expansion up to $z$ is accelerating or decelerating on average, not the state of acceleration at a given redshift.

We therefore turn to the second common claim, namely that if the difference $\Delta m$ increases with redshift, the universe is accelerating at that very redshift. The derivative of $\Delta m$ with respect to redshift is given by

$$
(\Delta m)^{\prime}=\frac{5}{\ln 10}\left(\frac{d_{L}^{\prime}}{d_{L}}-\frac{d_{L, n}^{\prime}}{d_{L, n}}\right) .
$$

We note that $(\Delta m)^{\prime}$ is independent of $H_{0}$ and $M$. For $(\Delta m)^{\prime}>0$, we have

$$
\frac{d_{L}^{\prime}}{d_{L}}>\frac{d_{L, n}^{\prime}}{d_{L, n}} .
$$




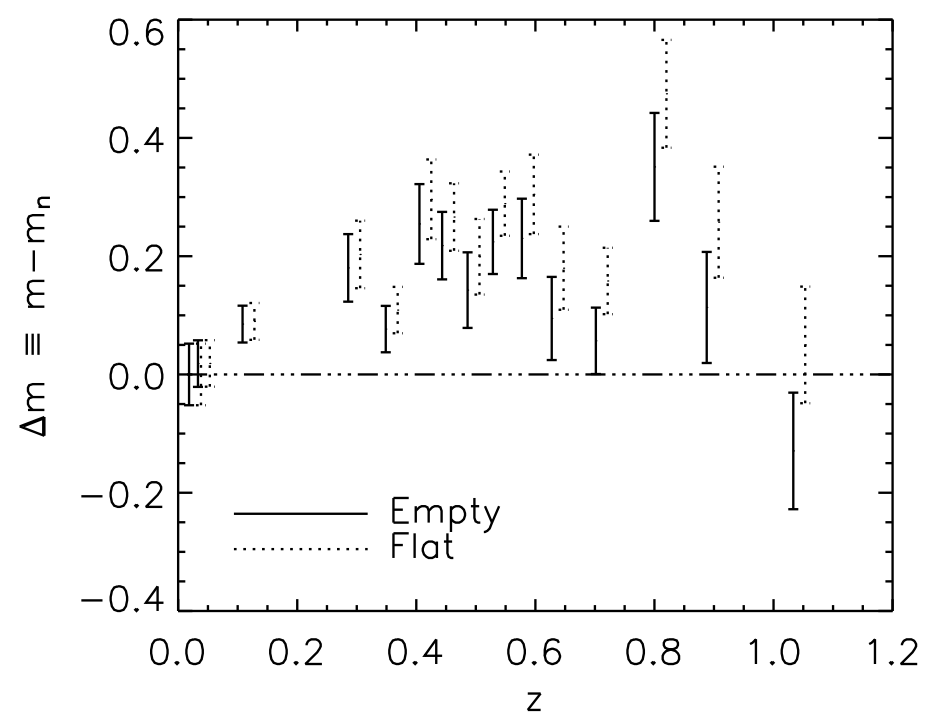

Figure 3. The differences between observed SN Ia magnitudes and the expected magnitudes for empty and flat non-accelerating universes. The differences are normalised to be zero at low redshifts. Error bars correspond to $68.3 \%$ CLs.

Assuming a flat universe, this amounts to

$$
H(z) \int_{0}^{z} \frac{d z}{H(z)}<(1+z) \ln (1+z) .
$$

From Eq. (25), it is evident that $(\Delta m)^{\prime}$ does not depend only on the state of acceleration at the given redshift, but also on the integral of the expansion. The sign of $(\Delta m)^{\prime}$ is thus not directly related to the state of acceleration. As a specific example, consider the concordance model with $\Omega_{m}=0.3$ and $\Omega_{\Lambda}=0.7$ in Fig. 4. The lines corresponding to $(\Delta m)^{\prime}$ and the deceleration parameter does not cross the zero line at the same redshift. We conclude that visually judging the state of the universal acceleration from a SN Ia Hubble diagram is non-trivial.

\subsection{Piecewise constant $q(z)$}

We divide our redshift range in $\left[z_{0}=0, z_{1}, z_{2}, z_{3}, \ldots\right]$, where between redshift, $z_{i-1}$ and $z_{i}$, we have a constant deceleration parameter $q_{i}$. In that bin, we then have $H(z) \propto(1+z)^{q_{i}+1}$. Since the normalisation of the Hubble parameter is marginalised over, we can put $H\left(z_{0}\right)=H_{0}=1$. For $0<z<z_{1}$, we then have $H(z)=(1+z)^{q_{1}+1}$, for $z_{1}<z<z_{2}$, we have $H(z)=\left(1+z_{1}\right)^{q_{1}-q_{2}}(1+z)^{q_{2}+1}$, etc.

The simplest case of a piecewise constant $q(z)$ would be to have a single constant value of the deceleration parameter, i.e., $q(z)=q_{0}$ and $H(z)=H_{0}(1+z)^{q_{0}+1}$, at all times. Actually, such a simple model gives a reasonable fit to SN Ia data, especially when allowing for curvature, see left panel of Fig. 5. To fit the CMB and BAO data 


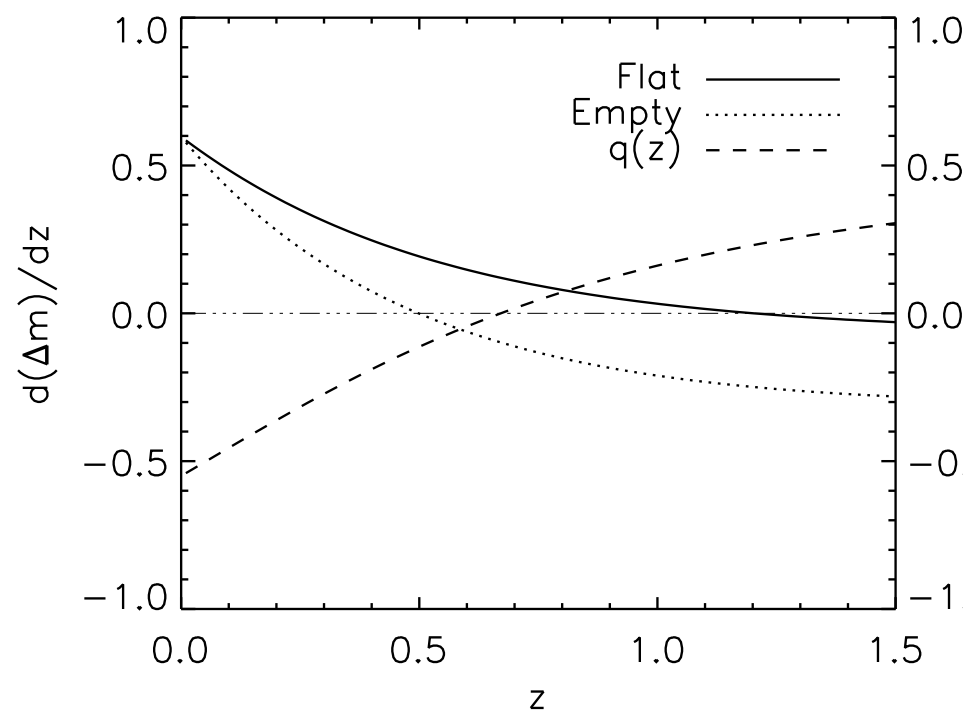

Figure 4. $(\Delta m)^{\prime}=d(\Delta m) / d z$ compared to $q(z)$ for the concordance model. The solid and dotted lines shows the derivative of the difference between the concordance and the flat and empty non-accelerating models, respectively. There is not a one-to-one correspondence between the sign of $(\Delta m)^{\prime}$ and the state of acceleration.

using this simple model however, requires a large amount of fine-tuning since the quality of the fit depends very sensitively on the exact values of $\Omega_{k}$ and $q_{0}$. Also, since CMB and BAO prefers a close to flat solution, the model provides a poor fit to all data combined.

We next turn to an analysis along the lines of Turner and Riess [44] where we assume that the deceleration parameter $q(z)$ has one constant value at $z<z_{t}$ and one constant value at $z>z_{t}$. We first choose $z_{t}=0.7$ in order to maximise our chances to detect a difference in the change of the expansion rate, since this is the transition redshift for the concordance cosmology that we know provides a good fit to the data. Results using SN Ia data are shown in the right panel of Fig. 5. Allowing for curvature increases the size of the contours by a factor of $\sim 2$. Incorporating also CMB and BAO data has the effect of constraining the curvature down to the level where the combined results are very similar to SN Ia results when assuming zero curvature, except for a tail of very low values for $q(z>0.7)$ if the universe has a small positive curvature $\left(\Omega_{k}<0\right.$, see Fig. 9). Note that the data does not show any evidence for deceleration at $z>0.7$. However, the evidence for acceleration at $z<0.7$ is very strong; $\sim 5 \sigma$ for SN Ia data only and $\sim 7 \sigma$ including $\mathrm{CMB}$ and BAO data or assuming a flat universe. However, as shown in Shapiro and Turner [43], marginalising over the transition redshift, considerably relaxes the constraints on the expansion history. In the left panel of Fig. 6, we show how the confidence contours change with the transition redshift, $z_{t}$, assuming a flat universe. For high $z_{t}$, results are mostly sensitive to $q\left(z<z_{t}\right)$, and vice versa. In the right panel of Fig. 6, results when marginalising over the transition redshift 

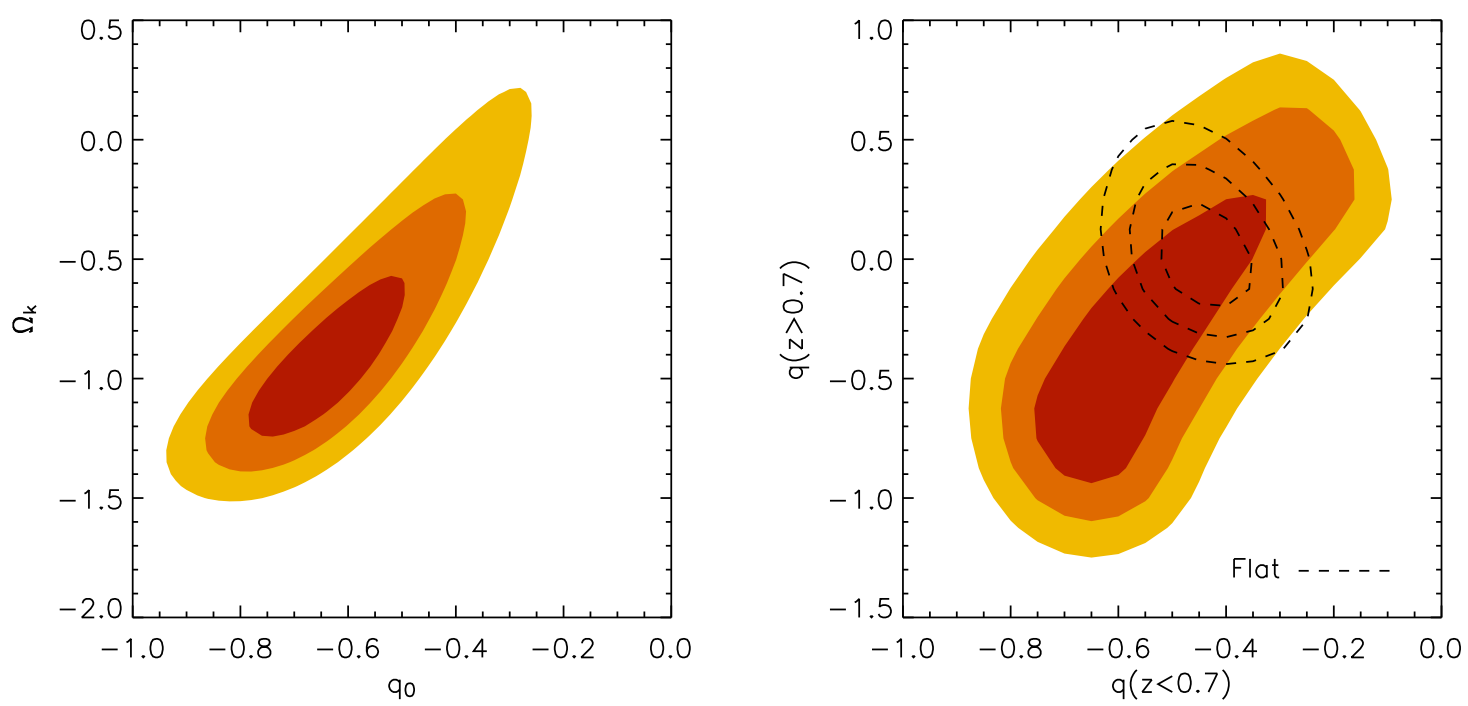

Figure 5. Left panel: SN Ia constraints on a model where the deceleration parameter has a constant value $q(z)=q_{0}$. Right panel: SN Ia constraints on a model where the deceleration parameter $q$ has one constant value at $z<0.7$ and one constant value at $z>0.7$. Allowing for curvature increases the size of the contours by a factor of $\sim 2$. The evidence for acceleration at $z<0.7$ is $\sim 7 \sigma$ and $\sim 5 \sigma$, respectively. Contours correspond to $68.3 \%, 95.4 \%$ and $99.7 \%$ CLs.

in the interval $0.1<z_{t}<1.0$ are shown, with and without the assumption of a flat universe. The upper, right quadrant, corresponding to expansion histories without any acceleration, can be ruled with high confidence, independent of curvature. However, all other possibilities are still viable, including deceleration at low redshifts and acceleration at higher redshifts, if the transition redshift is low enough $\left(z_{t} \sim 0.1\right)$.

Next, we allow for a finer redshift resolution in $q(z)$. Note that the $q_{i}$ are correlated, but we can decorrelate the $q_{i}$ estimates by (following Ref. [22]) changing the basis through an orthogonal matrix rotation that diagonalises the covariance matrix. This corresponds to applying a weight function to the $q_{i}$ to obtain decorrelated $Q_{i}$. These are linear combinations of $q_{i}$ where the weight function quantifies the redshift dependence of $Q_{i}$. First, we use SN Ia data only to constrain $q(z)$ in bins $z=[0 ., 0.3,0.6,1 ., 1.8]$, assuming a flat universe. Results for $q_{i}$ and the decorrelated $Q_{i}$ together with the corresponding weights are presented in Fig. 7. It is interesting to note that we again detect acceleration at low redshift with high confidence, but that the data do not require deceleration at higher redshifts. Allowing for curvature increases the error bars on $q(z)$ somewhat, but does not change our qualitative results.

Including $\mathrm{CMB}$ and $\mathrm{BAO}$ data, we can extend our analysis all the way out to $z \sim 1100$ in redshift bins $z=[0 ., 0.5,1 ., 1.8,1100$.$] . We can see (Fig. 8) that in the$ redshift interval $1.8<z<1100$, we in fact do see that the expansion was decelerating, assuming a flat universe. From the weight function, we can also see that the deceleration 

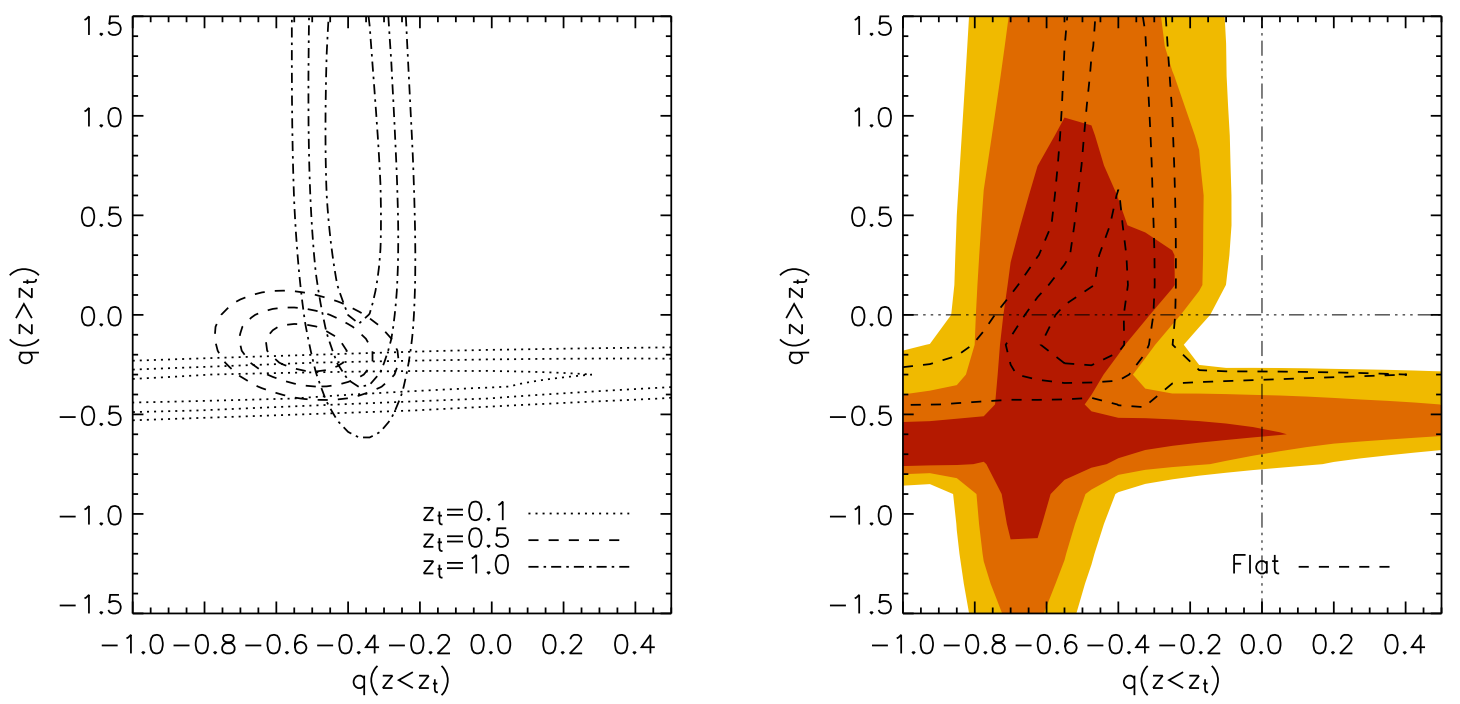

Figure 6. SN Ia constraints the deceleration parameter $q$ when varying the transition redshift $z_{t}$. In the left panel, confidence contours for three discrete values of $z_{t}$ in a flat universe is shown. In the right panel, results when marginalising over the transition redshift in the interval $0.1<z_{t}<1.0$ are shown, with and without the assumption of a flat universe. Contours correspond to $68.3 \%, 95.4 \%$ and $99.7 \%$ CLs.
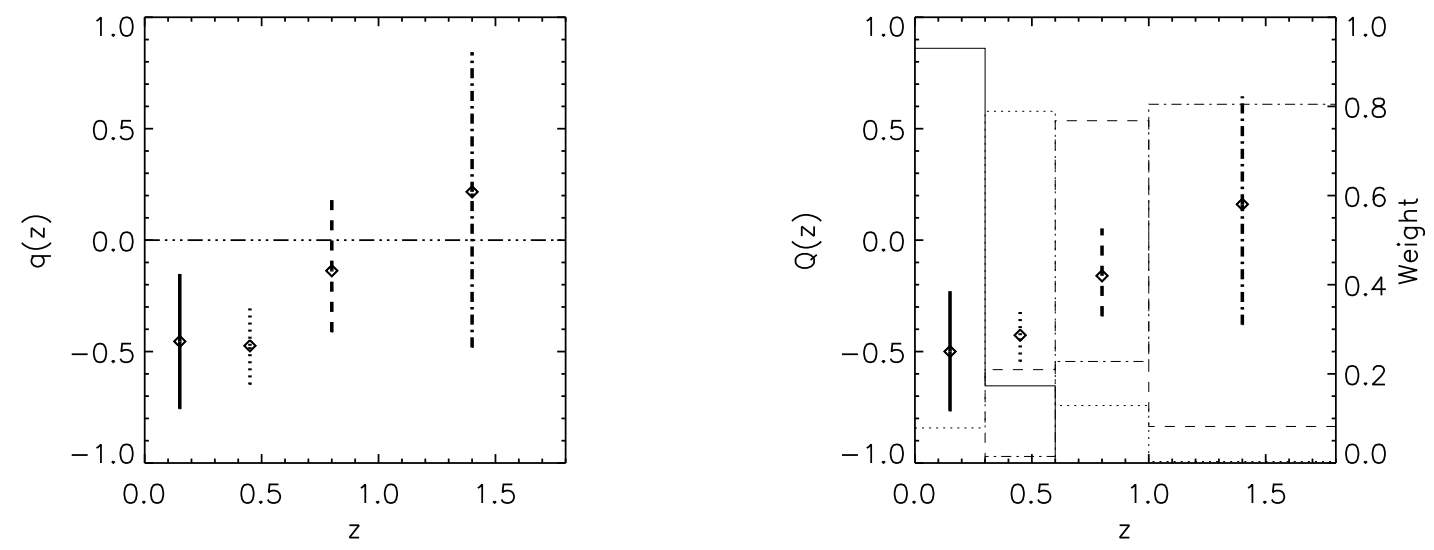

Figure 7. SN Ia constraints on $q(z)$ in bins $z=[0 ., 0.3,0.6,1 ., 1.8]$, assuming a flat universe. The left panel shows results for $q_{i}$, and the right panel decorrelated $Q_{i}$ together with the corresponding weights. Error bars represent $95.4 \%$ CLs. 

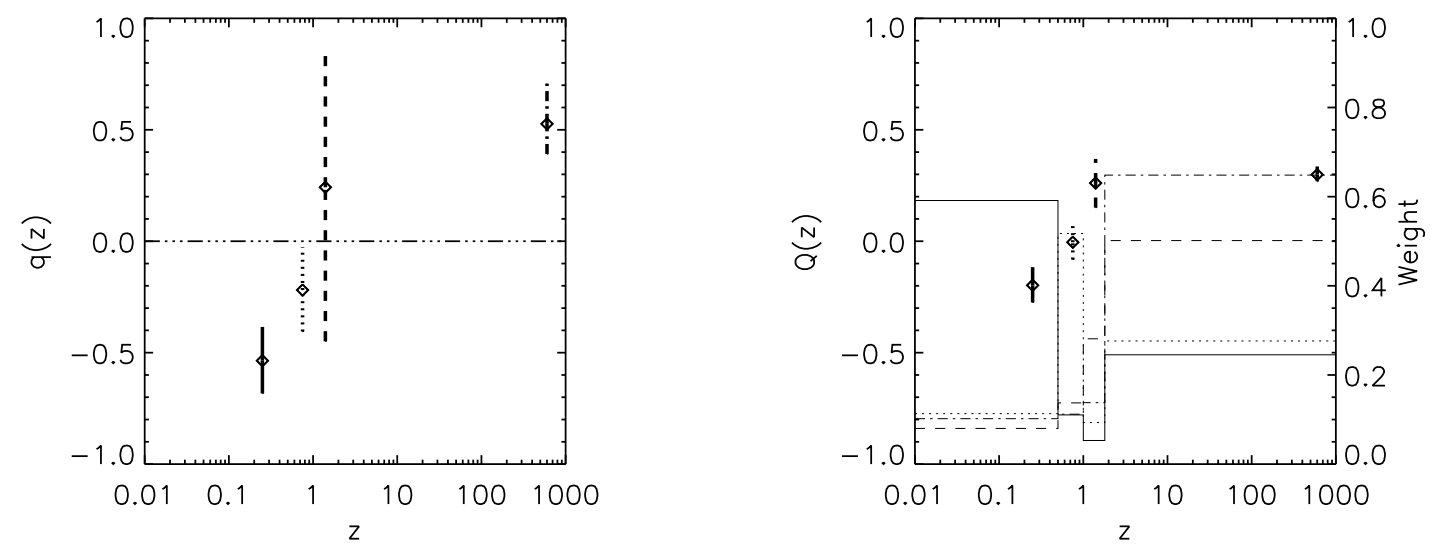

Figure 8. Constraints on $q(z)$ in redshift bins $z=[0 ., 0.5,1 ., 1.8,1100$.$] combining$ $\mathrm{SN}$ Ia, CMB and BAO data, assuming a flat universe. The left panel shows results for $q_{i}$, the right panel decorrelated $Q_{i}$ and the corresonding weight functions. In the redshift interval $1.8<z<1100$, the expansion is decelerating at high CL. Error bars represent $95.4 \%$ CLs.

parameter at different redshifts are very much correlated. If we relax the assumption of flatness, our Monte Carlo Markov Chains fail to converge, the reason being that the curvature and the deceleration parameter at high redshifts exhibit very interesting degeneracies, that also allow for solutions with high redshift acceleration and positive curvature. This degeneracy is easy to understand when considering the two component model where the deceleration parameter $q(z)$ has one constant value at $z<z_{t}$ and one constant value at $z>z_{t}$. For $z_{t}>0.35$, and a fixed $q\left(z<z_{t}\right)$, BAO constraints on $D_{V}$ only has a very weak (sub-percent) dependence on curvature, and the ratio between the scale of the BAO and CMB solely depends on the angular diameter distance to $z_{*} \sim 1090$

$$
d_{A}\left(z_{*}\right)(1+z *)=\frac{1}{H_{0} \sqrt{-\Omega_{k}}} \sin \left[\sqrt{-\Omega_{k}} H_{0} d_{c}\left(z_{*}\right)\right] .
$$

In the left panel of Fig. 9, we have plotted the difference between the measured and theoretical values of $d_{A}\left(z_{*}\right)$ for a fixed value of $D_{V}(z=0.2)$, normalised with the error on $d_{A}\left(z_{*}\right)$. The characteristic degeneracy structure arises because of the sinusoidal form of the angular distance in Eq. 28) and allows for models with high redshift acceleration and positive curvature to fit the data. In the right panel, the full confidence contours for SN Ia, CMB and BAO data are shown. It is evident that negative values for $q(z>0.7)$, i.e., corresponding to acceleration, are allowed if the universe has a small positive curvature. We note however, that such solutions require a considerable amount of fine tuning of the deceleration and curvature parameters. 

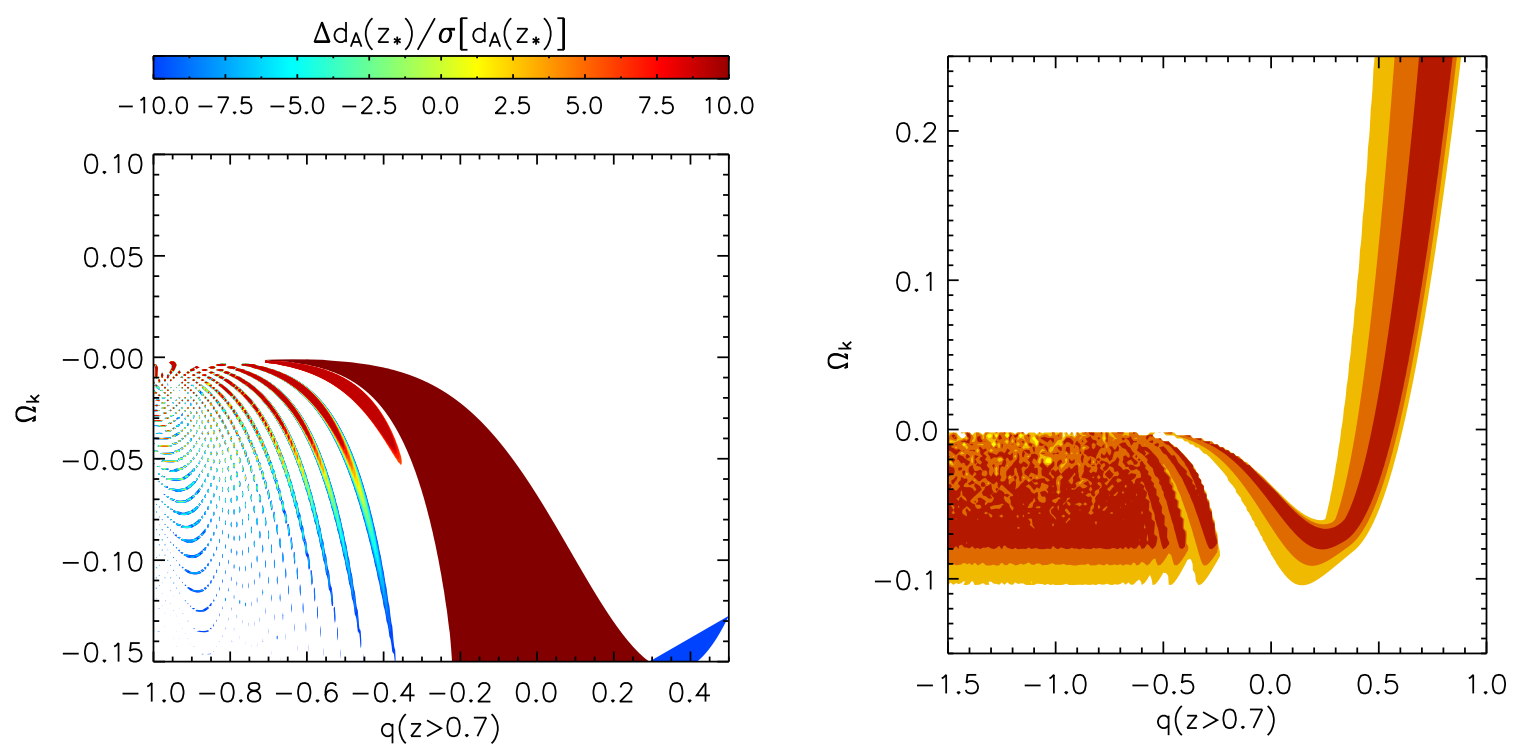

Figure 9. An example of the strong degeneracy between the curvature and the deceleration parameter at high redshifts in the simple model where $q(z)$ has one constant value at $z<0.7$ and one constant value at $z>0.7$. In the left panel, the difference between the measured value and theoretical values of $d_{A}\left(z_{*}\right)$ for a fixed value of $D_{V}(z=0.2)$, normalised with the measured error on $d_{A}\left(z_{*}\right)$, is shown. In the right panel, full confidence contours employing SN Ia, CMB and BAO data are shown. The degeneracy is not sensitive to the exact redshift binning of $q(z)$. The shaded areas represent $68.3 \%, 95.4 \%$ and $99.7 \%$ CLs.

\subsection{Parameterising $q(z)$}

There are many ways to parameterise $q(z)$. Clearly, a piecewise constant function as used above only gives a limited amount of information about $q(z)$; its average value over a certain redshift range. Ideally, we would prefer something which can closely mimic what the 'true' $q(z)$ might be up to. As an example, let us consider

$$
q(a)=q_{0}+q_{a}(1-a)=q_{0}+q_{a} \frac{z}{1+z},
$$

adopted from one of the most common parameterisation of the dark energy equation of state, $w(z)[7]$. At zero redshift, $q(a=1)=q_{0}$ and in the infinite past, $q(a=0)=q_{0}+q_{a}$. This parameterisation appears to be reasonably flexible in the sense that performing a least squares fit to many random dark energy models, always provides an acceptable fit. In Fig. 10, we show results for this parameterisation, both with and without curvature. Once again curvature degrades all constraints, though not as significantly as for other models for the SN Ia constraints on $q(z)$. Therefore, fitting SN Ia data alone using this parameterisation, we are able to conclude that the universe is accelerating today at $>5 \sigma$, irrespective of curvature.

For $q_{a}=0$, the model has $q(z)=q_{0}$ and is equivalent to the model presented in Fig. 5. Studying the contours at $q_{a}=0$ in Fig. 10, it is again obvious that this simple 

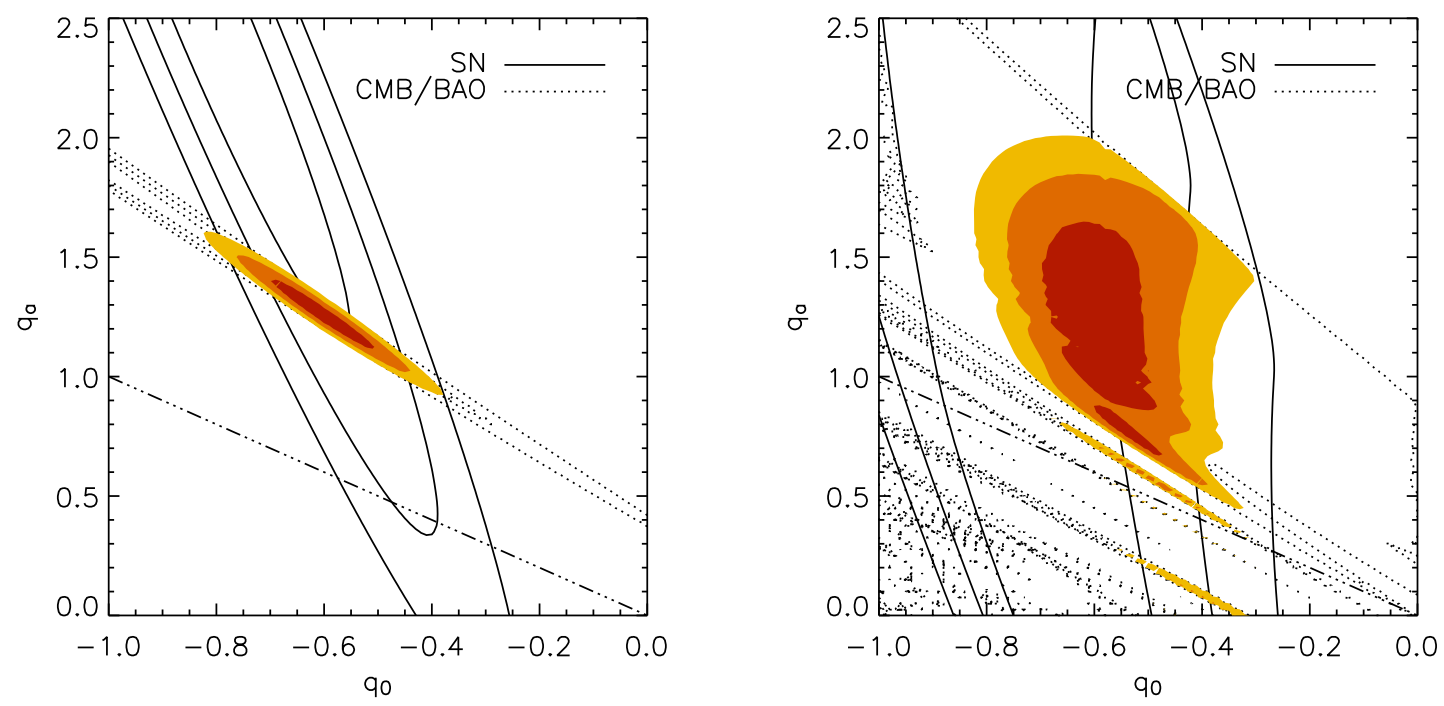

Figure 10. Constraints on $q_{0}, q_{a}$ for the parameterisation given by Eq. 29], assuming a flat universe (left) and allowing for curvature (right). The dash-dotted line is given by $q_{0}+q_{a}=0$, and separates regions with eternal acceleration (lower left) from regions with past deceleration (upper right). Using only SN Ia data, $q_{0}<0$ at $>5 \sigma \mathrm{CL}$, irrespective of the spatial curvature. The degenerate structures in the right panel are discussed in Sec. 4.2. Regions represent $68.3 \%, 95.4 \%$ and $99.7 \%$ CLs.

model does not provide a good fit to the data, unless we allow for curvature and an extreme fine-tuning of the parameters of the model.

\subsection{The Hubble parameter}

Since it is possible, although difficult (see Fig. 3), to obtain the Hubble parameter by differentiating distances, which would give us $q(z)$ directly, we can try to compare $\dot{a}^{-1}=(1+z) / H(z)$ at different redshifts [35]. We follow the technique proposed in Wang and Tegmark [47] to extract the expansion history in uncorrelated redshift bins from SN Ia data. The results are shown in Fig. 11, where increasing values of $(1+z) / H(z)$ corresponds to acceleration and vice versa. Unfortunately, because of the differentiation of sparse and noisy data, results are quite sensitive to the employed binning of the data, especially at high redshift. In the left panel of Fig 11, redshifts bins of size $\Delta z=0.3$ has been used whereas in the right panel, $\Delta z=0.25$. It is therefore difficult to assess the exact CL for the trend seen in Fig. 11 of increasing $(1+z) / H(z)$ - corresponding to acceleration - at low redshifts and decreasing $(1+z) / H(z)$ - corresponding to deceleration - at high redshifts. However, independent of the bin size, acceleration at low redshifts seems to be inevitable whereas deceleration at higher redshifts is not. 

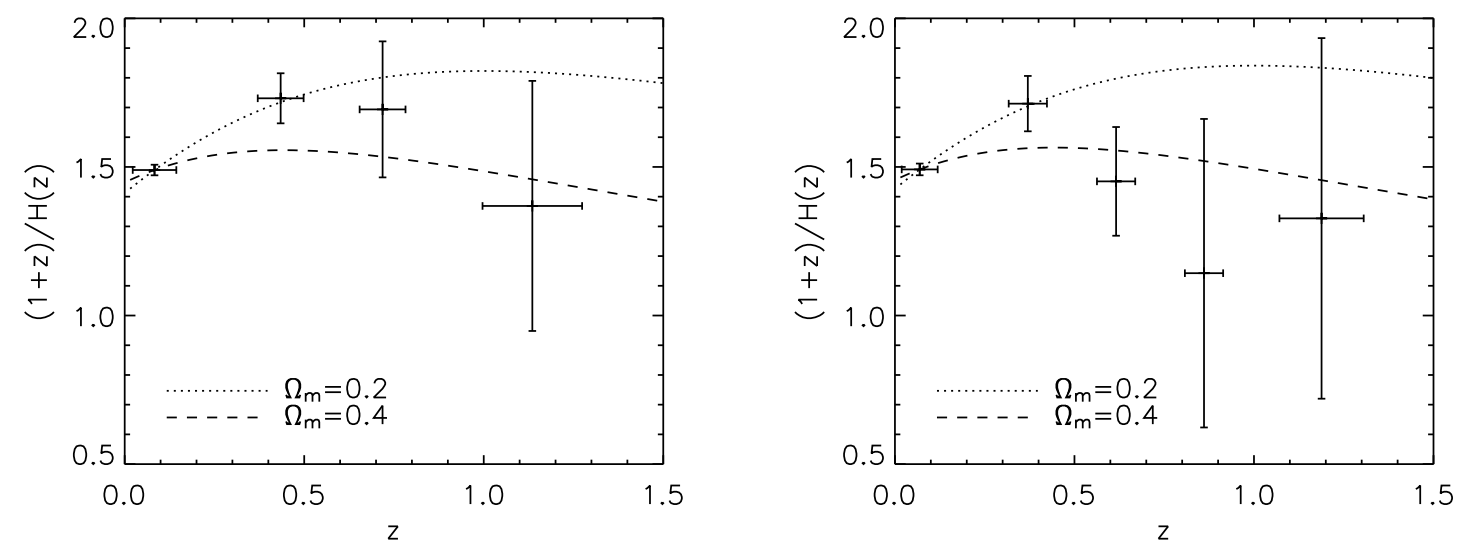

Figure 11. SN Ia constraints on $\dot{a}^{-1}=(1+z) / H(z)$. Values increasing with redshift correspond to acceleration and vice versa. The dotted lines correspond to a flat universe with $\Omega_{m}=0.2$ and $\Omega_{m}=0.4$ from top to bottom. The normalisation is arbitrary and is set to agree at low redshifts. In the left panel of Fig 11, redshifts bins of size $\Delta z=0.3$ has been used whereas in the right panel, $\Delta z=0.25$. Vertical error bars correspond to $68.3 \%$ CLs. Acceleration at low redshifts is clearly detected whereas deceleration at higher redshifts is not.

\subsection{Sliding Taylor expansion}

We can Taylor expand the scale factor around the current value according to

$$
\begin{aligned}
a(t) & =a_{0}+\dot{a}_{0}\left(t-t_{0}\right)+\frac{1}{2} \ddot{a}_{0}\left(t-t_{0}\right)^{2}+\mathcal{O}\left(t-t_{0}\right)^{3} \\
& =1+H_{0}\left(t-t_{0}\right)-\frac{1}{2} q_{0} H_{0}^{2}\left(t-t_{0}\right)^{2}+\mathcal{O}\left(t-t_{0}\right)^{3} .
\end{aligned}
$$

Since the comoving coordinate distance is given by

$$
d_{c}\left(t_{0}\right)=\int_{t_{e}}^{t_{0}} \frac{d t}{a(t)}
$$

we can write

$$
d_{c}(z) \simeq \frac{z}{H_{0}}\left[1-\frac{1+q_{0}}{2} z\right]
$$

To first order, the distance is given by the expansion rate of the universe today, or $H_{0}$, and to second order by the change in the expansion rate today, or $q_{0}$. We now generalise Eq. (33) to allow for a Taylor expansion around any time, or equivalently, redshift according to

$$
\begin{aligned}
a(t) & \simeq a\left(t_{1}\right)+\dot{a}_{1}\left(t-t_{1}\right)+\frac{1}{2} \ddot{a}_{1}\left(t-t_{1}\right)^{2} \\
& =a_{1}\left[1+H_{1}\left(t-t_{1}\right)-\frac{1}{2} q_{1} H_{1}^{2}\left(t-t_{1}\right)^{2}\right]
\end{aligned}
$$

The comoving coordinate distance is given by

$$
d_{c}\left(t_{0}\right) \simeq \frac{1}{a_{1}}\left[\left(t_{0}-t_{1}\right)-\frac{H_{1}}{2}\left(t_{0}-t_{1}\right)^{2}-\left(t_{e}-t_{1}\right)+\frac{H_{1}}{2}\left(t_{e}-t_{1}\right)^{2}\right],
$$




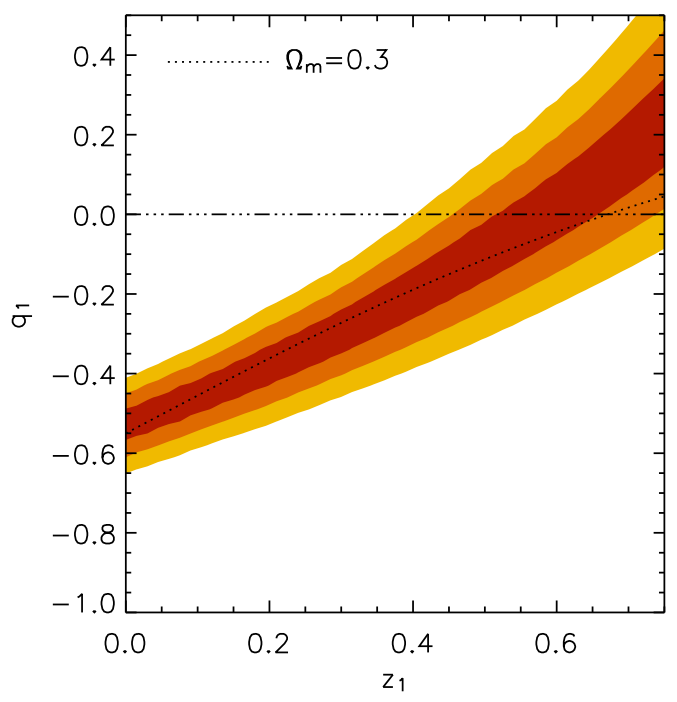

Figure 12. Fitting the deceleration parameter, $q\left(z_{1}\right)=q_{1}$, with a sliding expansion redshift for data simulated within a flat, cosmological constant universe with $\Omega_{m}=0.3$. The bias between the fitted $q_{1}$ and the true $q(z)$ (dotted line) caused by higher order terms in the Taylor expansion lies within the $68.3 \%$ CL for $z_{1} \lesssim 0.6$.

or, in terms of observables,

$$
d_{c}(z) \simeq \frac{z}{H_{1}}\left[1+\frac{z_{1}\left(1+q_{1}\right)}{1+z_{1}}-\frac{1}{2} \frac{\left(1+q_{1}\right)}{\left(1+z_{1}\right)} z\right] .
$$

We can now fit the parameter $q_{1}$, using SN Ia data for a sliding expansion redshift $z_{1}$, while marginalising over $H_{1}$. In Fig. 12 , we show results obtained using simulated data, corresponding to the Union08 data set, within the concordance cosmology. We only include SNe for which $\left|z-z_{1}\right|<0.75$ to guarantee convergence, which is why this and subsequent plots only extend to $z=0.75$. As can be seen in Fig. 12, the bias from higher order terms is within the $68.3 \%$ CL for $z \lesssim 0.6$ and within $95.4 \%$ CL all the way up to $z=0.75$.

In Fig. 13, results for the real Union08 data set, with and without curvature $\left(-1<\Omega_{k}<1\right)$, is shown. Positive $\Omega_{k}$ pushes $q(z)$ towards more positive values and vice cersa. Again, we only include data for which $\left|z-z_{1}\right|<0.75$ to reduce bias from higher order terms in the Taylor expansion.

Regardless of the expansion redshift, the sliding Taylor expansion is able to provide a very good fit to the SN Ia data. Note however that the results for $q_{1}$ at different redshifts are not independent, and it is therefore not possible to interpret the results as giving a full functional form of deceleration parameter $q(z)$. Assuming zero curvature, the universe can be shown to be accelerating at $z \lesssim 0.5$ and there is weak $(95.4 \%)$ evidence for decelaration at high redshifts. Including curvature severly degrades these constraints, especially the behaviour of $q(z)$ at high redshifts. Nevertheless, using the sliding Taylor expansion, the evidence for acceleration at $z=0$ is $>12 \sigma$, even when 

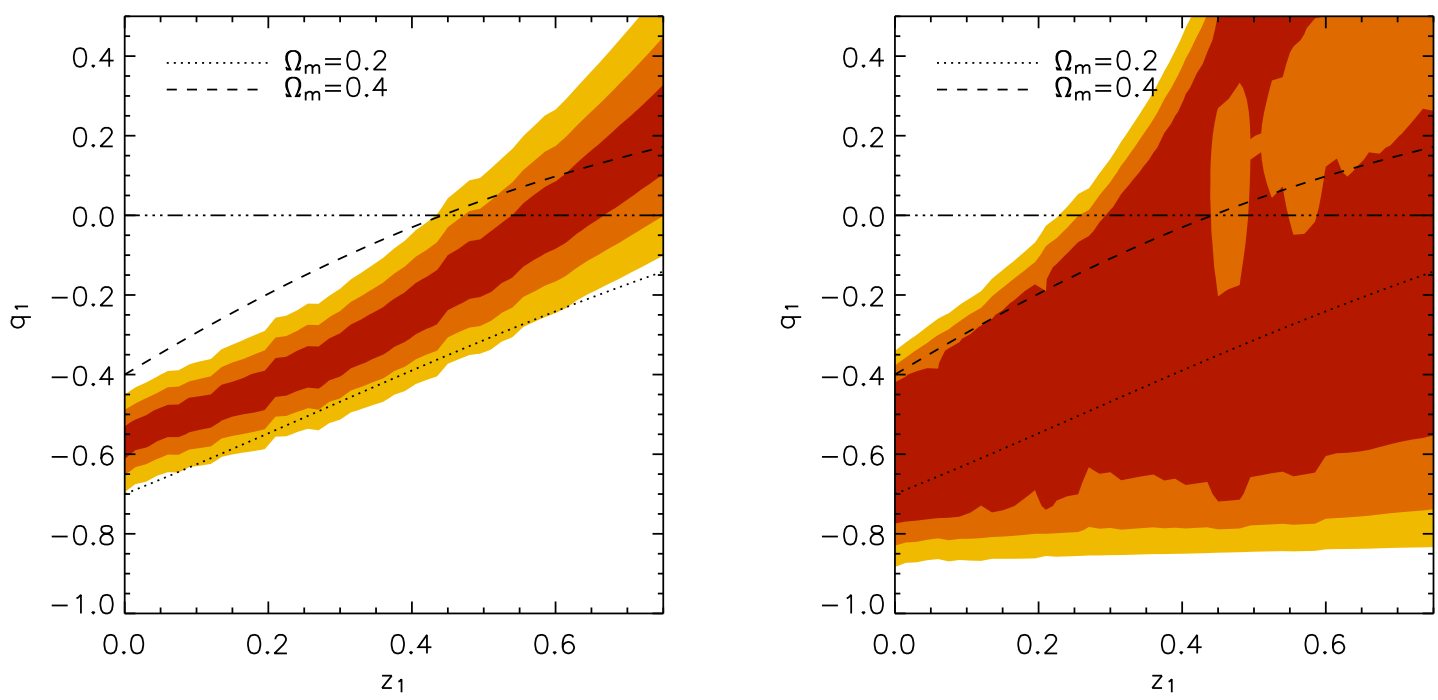

Figure 13. Fitting the deceleration parameter, $q\left(z_{1}\right)=q_{1}$, with a sliding expansion redshift. Note that constraints on $q_{1}$ are significantly looser when allowing for curvature (in this case $-1<\Omega_{k}<1$ ). However, the evidence for acceleration at $z=0$ is still $>12 \sigma$, including curvature. For a flat universe, $q_{1}\left(z_{1}=0\right)<0$ at $\sim 15 \sigma \mathrm{CL}$. The lines correspond to the flat, cosmological constant universes with $\Omega_{m}=0.2$ and 0.4 , respectively. The shaded areas correspond to $68.3 \%, 95.4 \%$ and $99.7 \%$ CLs.

allowing for curvature.

The reason for the closeness of the 2 and $3 \sigma$ contours in the right panel, is that at each redshift, the confidence contours in the $\left[\Omega_{k}, q_{1}\right]$-plane has a banana like shape. This has the effect that the resulting confidence levels for $q_{1}$, which are obtained by projecting the banana shaped contours on the $q_{1}$-axis, has a non-gaussian shape.

\section{Summary}

In this paper, we have investigated to what extent we can measure the change in the universal expansion rate, without making any assumptions about the energy content of the universe. Consequently we are limited to geometrical data as opposed to methods that are sensitive to the growth of structure in the universe. The data employed in this paper includes the redshift-distance relation of Type Ia SNe, as well as the the ratio of the scale of the baryon acoustic oscillations as imprinted in the cosmic microwave background and in the large scale distribution of galaxies. We have used several different methods to constrain the expansion history of the universe, all of which give the same qualitative result.

From SN Ia data alone, it is evident that the universal expansion is accelerating at low redshifts. In particular our new sliding expansion redshift method is able to detect acceleration today at $>12 \sigma$, even allowing for curvature. Although there are hints from SN Ia data that the universal expansion may have decelerated at high redshifts - as 
expected in the concordance cosmological model - we can only draw that conclusion with high confidence if we also include $\mathrm{CMB}$ and BAO data, together with the assumption of a flat or open universe. If the universe has a small positive curvature $\left(\Omega_{k}<0\right)$, it is possible, although it requires a certain level of fine tuning, to accommodate the data with acceleration also at high redshifts.

\section{Acknowledgments}

The authors would like to thank the anonymous referee for useful comments on the paper. EM acknowledge support for this study by the Swedish Research Council and SIDA. CC is funded by the NRF (South Africa). This was work initiated at a meeting funded by the SASWE - Cosmology Bilateral agreement between South Africa and Sweden.

\section{References}

[1] Astier, P., J. Guy, N. Regnault, et al., 2006, Astron. Astrophys., 447, 31

[2] Behrend, J., I. A. Brown, and G. Robbers, 2008, Journal of Cosmology and AstroParticle Physics, 1, 13

[3] Bolejko, K., in Golovin, A., G. Ivashchenko, and A. Simon, editors, 13th Young Scientists' Conference on Astronomy and Space Physics (2006)

[4] Bolejko, K. and J. S. B. Wyithe, 2008, ArXiv e-prints

[5] Buchert, T., 2008, General Relativity and Gravitation, 40, 467

[6] Caldwell, R. R. and A. Stebbins, 2008, Physical Review Letters, 100, 19, 191302

[7] Chevallier, M. and D. Polarski, 2001, International Journal of Modern Physics D, 10,213

[8] Clarkson, C., B. Bassett, and T. H.-C. Lu, 2008, Physical Review Letters, 101, 1, 011301

[9] Clarkson, C., M. Cortês, and B. Bassett, 2007, Journal of Cosmology and AstroParticle Physics, 8, 11

[10] Clifton, T., P. G. Ferreira, and K. Land, 2008, ArXiv e-prints

[11] Cole, S., W. J. Percival, J. A. Peacock, et al., 2005, MNRAS, 362, 505

[12] Coley, A. A., 2007, ArXiv e-prints

[13] Copeland, E. J., M. Sami, and S. Tsujikawa, 2006, International Journal of Modern Physics D, 15, 1753

[14] Daly, R. A., S. G. Djorgovski, K. A. Freeman, et al., 2008, Astrophys. J., 677, 1

[15] Davis, T. M., E. Mörtsell, J. Sollerman, et al., 2007, Astrophys. J., 666, 716

[16] Eisenstein, D. J., I. Zehavi, D. W. Hogg, et al., 2005, Astrophys. J., 633, 560

[17] Elgarøy, Ø. and T. Multamäki, 2006, Journal of Cosmology and Astro-Particle Physics, 9, 2 
[18] Garcia-Bellido, J. and T. Haugboelle, 2008, ArXiv e-prints

[19] Garcia-Bellido, J. and T. Haugbølle, 2008, Journal of Cosmology and Astro-Particle Physics, 4, 3

[20] García-Bellido, J. and T. Haugbølle, 2008, Journal of Cosmology and Astro-Particle Physics, 9, 16

[21] Hlozek, R., M. Cortês, C. Clarkson, and B. Bassett, 2008, General Relativity and Gravitation, 40, 285

[22] Huterer, D. and A. Cooray, 2005, Physical Review D, 71, 2, 023506

[23] Jönsson, J., T. Dahlén, A. Goobar, et al., 2006, Astrophys. J., 639, 991

[24] Komatsu, E., J. Dunkley, M. R. Nolta, et al., 2008, ArXiv e-prints, 803

[25] Kowalski, M., D. Rubin, G. Aldering, et al., 2008, ArXiv e-prints, 804

[26] Larena, J., J.-. Alimi, T. Buchert, M. Kunz, and P.-S. Corasaniti, 2008, ArXiv e-prints

[27] Li, N. and D. J. Schwarz, 2007, Physical Review D, 76, 8, 083011

[28] Li, N., M. Seikel, and D. J. Schwarz, 2008, ArXiv e-prints

[29] Percival, W. J., S. Cole, D. J. Eisenstein, et al., 2007, MNRAS, 381, 1053

[30] Perlmutter, S., G. Aldering, G. Goldhaber, et al., 1999, Astrophys. J., 517, 565

[31] Rapetti, D., S. W. Allen, M. A. Amin, and R. D. Blandford, 2007, MNRAS, 375, 1510

[32] Räsänen, S., 2008, Journal of Cosmology and Astro-Particle Physics, 4, 26

[33] Rasanen, S., 2008, ArXiv e-prints

[34] Riess, A. G., A. V. Filippenko, P. Challis, et al., 1998, Astron. J., 116, 1009

[35] Riess, A. G., L.-G. Strolger, S. Casertano, et al., 2007, Astrophys. J., 659, 98

[36] Riess, A. G., L.-G. Strolger, J. Tonry, et al., 2004, Astrophys. J., 607, 665

[37] Rosenthal, E. and É. É. Flanagan, 2008, ArXiv e-prints

[38] Sarkar, S., 2008, General Relativity and Gravitation, 40, 269

[39] Schmidt, B. P., N. B. Suntzeff, M. M. Phillips, et al., 1998, Astrophys. J., 507, 46

[40] Seikel, M. and D. J. Schwarz, 2008, Journal of Cosmology and Astro-Particle Physics, 2, 7

[41] Seikel, M. and D. J. Schwarz, 2008, ArXiv e-prints

[42] Seo, H.-J. and D. J. Eisenstein, 2003, Astrophys. J., 598, 720

[43] Shapiro, C. and M. S. Turner, 2006, Astrophys. J., 649, 563

[44] Turner, M. S. and A. G. Riess, 2002, Astrophys. J., 569, 18

[45] Uzan, J.-P., C. Clarkson, and G. F. R. Ellis, 2008, Physical Review Letters, 100, 19, 191303

[46] Vanderveld, R. A., É. É. Flanagan, and I. Wasserman, 2008, Physical Review D, $78,8,083511$ 
[47] Wang, Y. and M. Tegmark, 2005, Physical Review D, 71, 10, 103513

[48] Zibin, J. P., 2008, Physical Review D, 78, 4, 043504 